\title{
Gemeinsame Entschließung des Treffens der europäischen Informationsfreiheitsbeauftragten vom 24. Februar 2017
}

Nach zweitägiger Tagung in Berlin endet das Treffen der Europäischen Informationsfreiheitsbeauftragten und -ombudsleute am 24. Februar 2017 mit einem Schulterschluss: In einer gemeinsamen Entschließung bekennen sie sich zu einer europaweiten Durchsetzung der Informationsfreiheit und korrespondierend dazu zur nachhaltigen Stärkung ihrer Position als Mediator zwischen Staat und Gesellschaft. Dazu appellieren sie an Parlamente und Regierungen in Europa, bei denen noch keine Informationsfreiheitsbeauftragten das Recht auf Informationszugang durchsetzen, diese wichtige Institution zu etablieren und ausreichend personell und finanziell auszustatten. Mit der steigenden Bedeutung der Informationsfreiheit steigt auch das Bedürfnis der Bürgerinnen und Bürger nach Unterstützung, diesem Recht Geltung zu verleihen. Dazu müssen die Informationsfreiheitsbeauftragten über die erforderliche Ausstattung und die erforderlichen Kompetenzen verfügen.

Der Landesbeauftragte für die Informationsfreiheit Rheinland-Pfalz Prof. Dr. Dieter Kugelmann, der derzeit den Vorsitz der Konferenz der Informationsfreiheitsbeauftragten in Deutschland innehat, legt dabei ein besonderes Augenmerk auf die europäische Harmonisierung und regt an: „Informationsfreiheit ist Ausdruck eines modernen Demokratie- und Staatsverständnisses, das europaweit gelebt wird. Für eine effektive Gewährleistung braucht Europa gemeinsame Standards der Informationsfreiheit. Diese sollten im konstruktiven Dialog der Beauftragten, Ombudsleute, Parlamente und Regierungen erarbeitet werden.“ Die Weltkonferenz der Informationsfreiheitsbeauftragten diesen September in Manchester wird weitere Gelegenheit für einen internationalen Austausch geben.

\section{Entschließung der Europäischen Informationsfreiheitsbeauftragten und-ombudsleute ${ }^{1}$}

Informationsfreiheit durchsetzen! Europäische Informationsfreiheitsbeauftragte stärken!

1 Mit Blick auf das Mandat der europäischen Bürgerbeauftragten, der dänischen parlamentarischen und der norwegischen parlamentarischen Ombudsperson haben sich diese nicht an der Entschließung beteiligt.
Zahlreiche Staaten und Regionen in Europa haben den Anspruch auf Zugang zu Verwaltungsinformationen gesetzlich geregelt. Informationsfreiheitsbeauftragte und -ombudsleute unterstützen und beraten die Bürgerinnen und Bürger bei der Wahrnehmung ihrer Informationsrechte. Gleichzeitig fördern sie die gesetzeskonforme Anwendung der Informationsfreiheitsrechte auch durch entsprechende Beratung und Kontrolle der Behörden.

Ein starkes Informationsfreiheitsrecht kann seine volle Wirkung aber nur dann entfalten, wenn seine Umsetzung durch starke Informationsfreiheitsbeauftragte und -ombudsleute als Mittler zwischen Staat und Bürgern begleitet und gefördert wird.

Die Teilnehmer des Europa-Meetings der nationalen und regionalen

Informationsfreiheitsbeauftragten und -ombudsleute fordern deshalb die Parlamente und Regierungen in Europa auf, sicherzustellen, dass alle Menschen in ihren Ländern das Recht haben, unabhängige Einrichtungen anzurufen und diesen eine der Bedeutung ihrer Aufgabe adäquate budgetäre, personelle und befugnisrechtliche Ausstattung zu gewähren, damit das Recht der Bürgerinnen und Bürger auf Informationszugang wirkungsvoll unterstützt und gestärkt wird. Denn Informationsfreiheit und Transparenz sind bedeutende Faktoren für eine funktionierende freiheitliche und demokratische Rechtsordnung Informationsfreiheit in Europa lebt aber auch vom Austausch über Regelungsmodelle und Erfahrungen. Sie wird durch die Formulierung fundierter gemeinsamer Positionen der Informationsfreiheitsbeauftragten gerade auch zu Regelungsvorhaben auf supranationaler Ebene weiter gestärkt.

Die Arbeit des Netzwerkes europäischer Informationsfreiheitsbeauftragter und -ombudsleute soll daher z.B. durch regelmäßige Treffen verstetigt und weiter gestärkt werden.

Die Gruppe hat festgestellt, dass Europa gemeinsame Standards der Informationsfreiheit braucht. Diese sollten im konstruktiven Dialog der Beauftragten, Ombudsleute, Parlamente und Regierungen vorangebracht werden. 